COMMENT. The original reports of Williams (alt. Williams-Beuren) syndrome appeared in the journal Circulation, 1961 and 1962, with emphasis on "Supravalvular aortic stenosis." Diagnosis of Williams syndrome involves recognition of physical features and markers, followed by a confirmatory genetic test. This case report is presented as the first patient with WB syndrome to have a congenital CNS anomaly in addition to progressive hypertrophic cardiomyopathy. Neurologic symptoms previously reported include mild microcephaly, intellectual disability, and personality disorders (hyperverbal). Abnormalities in the cerebellum, right parietal cortex, and left frontal cortex are consistent with visual-spatial difficulties. Increased volume of the left auditory cortex correlates with a rhythm propensity and fondness of music. Neuropsychological, neurological, and neuroanatomical profile of Williams syndrome is compared to that of Down syndrome (Bellugi U et al. Am J Med Genet 1990;37(Suppl. S6):115-25). For a clinical guide to symptoms and diagnosis of Williams and other syndromes, see Millichap JG. Neurological Syndromes. New York: Springer; 2013. 279 p.

\title{
JOUBERT SYNDROME, A CILIOPATHY
}

Investigators at Neurogenetics Unit, Mendel Laboratory, Rome, and University of Salerno, Italy, review the clinical features and genetic basis of Joubert syndrome, overlap with other ciliopathies, and the multifaceted roles of primary cilia in CNS development. Joubert M. and colleagues first described a familial agenesis of the cerebellum, manifested by episodic hyperpnea, abnormal eye movements, ataxia and retardation (Neurology 1969 Sep;19(9):813-25). The characteristic malformation involving the cerebellum and brainstem, the MRI hallmark of the syndrome, is called the "molar tooth sign." Associated CNS defects include ventriculomegaly, meningo-encephalocele, polymicrogyria, periventricular nodular heterotopia, hypothalamic hamartoma, and corpus callosum defects. Specific Joubert syndrome subgroups are correlated with different causative genes, one known by the acronym $\mathrm{COACH}$ (cerebellar vermis hypoplasia, oligophrenia, ataxia, coloboma, and hepatic fibrosis). A total of 21 causative genes have been identified, all encoding for proteins of the primary cilium that has a key role in development. An increasing number of heterogeneous disorders are being causally related to mutations in ciliary genes. (Romani M, Micalizzi A, Valente EM. Joubert syndrome: congenital cerebellar ataxia with the molar tooth. Lancet Neurol 2013 Sep;12(9):894-905). (Response: Prof Enza Maria Valente: E-mail. e.valente@cssmendel.it).

COMMENT. Prenatal abnormal features of the fourth ventricle in fetuses with Joubert syndrome and related disorders are reported in 7 subjects, all showing the molar tooth sign using ultrasound and/or MRI. (Quarello E et al. Ultrasound Obstet Gynecol 2013 Jul 19 [Epub ahead of print]). The term "Joubert syndrome" now encompasses all molar tooth sign-related disorders, and the term "Joubert syndrome and related disorders" is no longer in favor. Variable clinical manifestations associated with the molar tooth sign are not distinct syndromes, but part of a wide phenotypic range characteristic of Joubert syndrome. 